\title{
GENETIC CONTROL OF INDUCTION OF ALKALINE PHOSPHATASE
}

SYNTHESIS IN E. COLI*

\author{
By A. Garen and H. Echols
}

BIOLOGY DIVISION, UNIVERSITY OF PENNSYLVANIA, AND BIOCHEMISTRY DEPARTMENT, UNIVERSITY OF WISCONSIN

Communicated by Seymour Benzer, May 28, 1962

Formation of the enzyme alkaline phosphatase in $E$. coli is under the genetic control of three genes. One of these, the $P$ gene, specifies the structure of the enzyme molecule. ${ }^{1-3}$ The other two genes, $R 1$ and $R 2$, regulate the rate of formation of the enzyme. In the wild-type strain $\left(P^{+} R 1^{+} R 2^{+}\right)$, the rate can vary over a thousandfold range, depending on the concentration of orthophosphate in the growth medium. The maximal rate is obtained when the concentration of orthophosphate is low; as the concentration is raised, the rate is reduced. ${ }^{4-6}$ The effect of a low concentration of orthophosphate on alkaline phosphatase formation is designated as induction, and that of a high concentration of orthophosphate as repression. Repression is jointly controlled by the $R 1$ and $R 2$ genes, as indicated by the isolation of constitutive (i.e., nonrepressible) mutants carrying mutations in either gene. ${ }^{7} \quad$ Induction appears to be under exclusive control of the $R 1$ gene, since mutations that cause a reduction in the rate of enzyme formation in low phosphate medium are known to occur in the $R 1$ gene, but not in the $R \mathscr{Q}$ gene.

Useful information about the functions of the $R 1$ and $R 2$ genes has been obtained from a study of heterozygous strains. With regard to repression, it was observed that if an $\mathrm{R1}^{-}$constitutive mutant was made heterozygous by the addition of a normal $R 1^{+}$gene, enzyme formation could be repressed. ${ }^{7}$ The same effect was found when a normal $R \mathscr{Q}^{+}$was added to an $R \mathscr{Q}^{-}$constitutive mutant. It was concluded from these results that repression was mediated by a cytoplasmic repressor, ${ }^{8}$ and that the production of the repressor depended upon the normal functioning of both $R 1$ and $R 2$ genes.

Induction was analyzed in an analogous way. Starting with an $R 1^{-}$constitutive mutant that could not produce the enzyme at the maximal rate in low-phosphate medium, it was found that this defect could be overcome by the addition of a normal $R 1^{+}$gene. ${ }^{9}$ This suggests that induction is mediated by a cytoplasmic inducer specified by the $R 1$ gene.

An explanation for these findings has recently been proposed. ${ }^{9}$ It assumes that the $R 1$ gene controls the formation of an endogenous inducer required for the synthesis of alkaline phosphatase and that the $R \mathscr{Z}$ gene controls the formation of an enzyme that can convert the inducer into a repressor when a high concentration of orthophosphate is present in the growth medium. This is illustrated by the following reaction scheme:

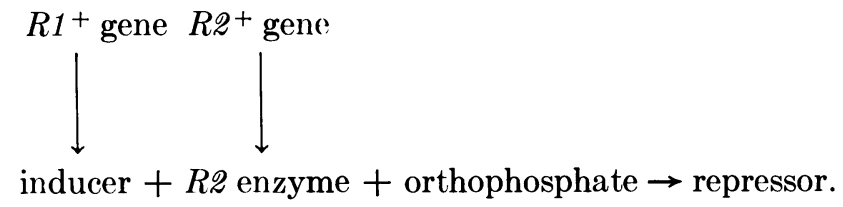

If an inducer is in fact involved in alkaline phosphatase synthesis, mutations should 
occur in the $R 1$ gene that completely block the formation of an active inducer. Such a mutation would generate a new type of phosphatase-negative mutant, one that maps in the $R 1$ gene rather than in the $P$ gene and shows complete complementation in tests against all $P^{-}$mutants. This type of mutant, which was first observed in the course of a study of complementation with phosphatase-negative mutants, ${ }^{10}$ is the subject of the present report.

Materials and Methods.-Bacterial strains: Heterozygous strains were produced by crosses between an $F^{\prime}$ donor and an $F^{-}$recipient. The $F^{\prime}$ contains an extrachromosomal segment, the episome, that is transferred to the $F^{-}$during mating. ${ }^{11}$ Strain $F^{\prime} 19$, isolated by Y. Hirota, was used in all experiments; the episome in this strain carries the $R 1$ and $P$ genes.

The $H f r$ used for all crosses was strain $K 10 .{ }^{7} \quad$ The selective markers $T^{+}, L^{+}$, and $S^{\mathrm{r}}$ refer, respectively, to growth in the absence of threonine and leucine and resistance to streptomycin.

Genetic crosses and enzymatic assays: The procedures have been described elsewhere. ${ }^{7,} 9$

Results.-Complementation behavior of phosphatase-negative mutants: The results of extensive complementation tests with phosphatase-negative mutants have been reported in a separate communication. ${ }^{10}$ The tests were run on trans heterozygotes produced with $F^{\prime}$ strains, in which one mutation was carried on the episome and another on the chromosome. Most of the mutations tested in this way gave clear evidence of belonging to the same cistron, since the trans heterozygotes involving all pairwise combinations between these mutations produced considerably less enzymatic activity than the wild-type strain. It has been shown that this cistron specifies the structure of the alkaline phosphatase enzyme. ${ }^{1-3}$

A few of the phosphatase-negative mutants that were analyzed in the complementation experiments behaved differently, producing a normal level of enzymatic activity in trans combinations with any of the mutants belonging to the structural gene. (The mutants showing this behavior are designated $C$ mutants, to distinguish them from the $P^{-}$mutants belonging to the structural cistron.) The results of a typical complementation test between $C$ and $P^{-}$mutants are given in Table 1.

TABLE 1

Complementation Tests with $P$ - and $C$ Mutants

\begin{tabular}{|c|c|c|c|}
\hline \multirow[b]{2}{*}{$\begin{array}{l}\text { Episoma } \\
\text { marker }\end{array}$} & \multirow[b]{2}{*}{$\begin{array}{c}\text { Chromosomal } \\
\text { marker }\end{array}$} & \multicolumn{2}{|c|}{ Alkaline Phosphatase Activity* } \\
\hline & & $\begin{array}{l}\text { Low phosphate } \\
\text { growth medium }\end{array}$ & $\begin{array}{l}\text { High phosphate } \\
\text { growth medium }\end{array}$ \\
\hline - & $\mathbf{P}^{+}$ & 100. & $<0.1$ \\
\hline - & $\mathbf{P}^{-}$ & $<0.1$ & $<0.1$ \\
\hline- & $\mathrm{C}$ & 0.2 & $<0.1$ \\
\hline $\mathbf{P}^{-}$ & $\mathbf{P}^{+}$ & 100. & $<0.1$ \\
\hline $\mathbf{P}^{-}$ & $\mathbf{C}$ & 110 . & $<0.1$ \\
\hline $\mathbf{P}+$ & $\mathbf{P}^{-}$ & $205 . \dagger$ & $<0.1$ \\
\hline $\mathbf{C}$ & $\mathbf{P}^{-}$ & 175. $\dagger$ & $<0.1$ \\
\hline
\end{tabular}

* Enzymatic activities are normalized to a standard cell density of the culture. * There appear to exist two copies of the episomal genes for each copy of the chromosomal genes in the $F^{\prime}$ strains,
which accounts for the twofold higher activity when the $P^{+}$gene is on the episome. ${ }^{+}$

It can be seen that the $C$ mutant complements with the $P$ - mutant to the same extent as does the wild-type, indicating that a normally functioning $P$ cistron is present in the $C$ mutant. The formation of phosphatase by the complementing heterozygotes is repressed in high-phosphate growth medium. When the comple- 
mentation test was applied to pairs of independently isolated $C$ mutants, involving four different (recombinable) sites, little or no increase in phosphatase activity was observed in the heterozygotes. Thus, $C$ and $P^{-}$mutations appear to involve two distinct cistrons, both of which control functions essential for the formation of alkaline phosphatase.

Mapping of $C$ mutations: The first cross was run to establish the map position of a $C$ mutation in relation to an $R 1^{-}$constitutive mutation. This was accomplished by crossing a $C$ mutant with an $R 1-P^{+}$mutant and examining the progeny for the presence of wild-type $\left(R 1^{+} P^{+}\right)$recombinants. None were found among the 670 progeny tested, indicating exceptionally close linkage $(<0.15$ recombination units) of the $R 1^{-}$and $C$ mutant sites.

The donor $H f r$ strain used in the following crosses transfers the $R 1$ and $P$ genes at 9 min after the start of mating, the $T$ and $L$ genes at $20 \mathrm{~min}$, and the $S$ gene sufficiently later so that the probability of its transfer is negligibly low. The $H f r$ parent in this first cross was a $C$ mutant with the selective markers $T^{+} L^{+} S^{\mathbf{s}}$, and the $F^{-}$parent was an $R 1^{-} P^{+}$mutant with the markers $T^{-} L^{-} S^{\mathrm{r}}$. Both parents carried an $\mathrm{R}^{+}$gene. The phosphatase character of cells was determined by plating either on high-phosphate (repressing) medium or on low-phosphate (nonrepressing) medium and then spraying the colonies with p-nitrophenyl phosphate (NPP). The $H f r$ parent scores negative (white colonies) and the $F^{-}$parent positive (yellow colonies) on both media. Any wild-type recombinants should score as negative on high-phosphate medium and as positive on low-phosphate medium. Mating was carried out as described under Methods, and the culture was plated on low-phosphate minimal medium containing streptomycin. Among the selected class of $T^{+} L^{+} S^{\mathrm{r}}$ colonies, about 60 per cent scored negative and 40 per cent positive by the NPP test, which indicates that the $C$ mutation is transferred before the $T$ and $L$ genes. The positive colonies, which might either be $R 1^{-} P^{+}$parental type or $R 1^{+} P^{+}$wildtype recombinants, were tested on high-phosphate medium. Among a total of 670 colonies examined, none were wild-type.

In a second cross, the $C$ mutation was mapped against a $P^{-}$mutation. The $C$ mutation was found to be located 1.8 recombination units from the $P^{-}$mutation, which is the same distance that separates an $R 1^{-}$mutation from the $P^{-}$mutation. ${ }^{7}$ This provides further evidence that the $C$ mutation lies within the region of the $R 1$ regulator gene.

The $H f r$ parent was the same $C$ mutant that was used in the preceding cross, and the $F^{-}$parent was an $R 1^{+} P^{-} R 2^{+} T^{-} L^{-} S^{\mathrm{r}}$ strain. Since the phosphatase character of both parents was negative, it was possible to score $P^{+}$recombinants selectively by plating on minimal streptomycin medium containing glycerol-phosphate as the sole source of phosphate. ${ }^{1,2}$ The mating procedure was the same as in the preceding cross. The proportion of $P^{+}$recombinants found among the selected class of $T^{+} L^{+} S^{\mathrm{r}}$ colonies was 1.8 per cent.

The final series of crosses show that $C$ mutations can occur at several sites, all of which are closely linked together. Six irdependently isolated $C$ mutants were crossed in various combinations. Most of the crosses yielded $P^{+}$recombinants, with recombination frequencies that were consistantly low $(<0.05$ recombination units).

Effect of $C$ mutations on repressor formation: The preceding mapping experiments 
have located the $C$ mutations in the $R 1$ gene. However, verification of this point requires a functional test to demonstrate that a $C$ mutation can affect the activity of the $R 1$ gene. For this purpose, $C$ mutants were tested for their'ability to produce a repressor of alkaline phosphatase. The experiments were carried out with $F^{\prime}$ heterozygotes that carried an $R 1^{-}$constitutive mutation on the episome and a $C$ mutation on the chromosome. The first two entries in Table 2 are controls show-

TABLE 2

Test for Repressor Formation by $C$ Mutants

$\begin{array}{cc}\text { Episomal markers } & \text { Chromosomal markers } \\ \mathrm{R}^{-} \mathrm{P}^{+} & \mathrm{R} 1^{-} \mathrm{P}^{-} \mathbf{R} 2^{+} \\ \mathrm{R} 1^{-} \mathrm{P}^{+} & \mathrm{R} 1^{+} \mathrm{P}^{-} \mathbf{R} 2^{+} \\ - & \mathrm{C} 1 \\ \mathrm{R}^{-} & \mathrm{C} 2 \\ \mathrm{R} 1^{-}-\mathrm{P}^{+} & \mathrm{C} 1 \\ \end{array}$

* The $R 1^{-}$constitutive mutation used for these strains is a type that prevents the maximal level of enzyme synthesis, ${ }^{7}$ which is the reason for activity being less than the value of 200 expected with a strain that carries a $P^{+}$gene on the episome.

ing that in an $R 1-P^{+} / R 1^{+} P^{-}$heterozygote, repressibility is dominant. Consequently, if a $C$ mutation impaired this $R 1$ function, the $R 1-P+/ C$ heterozygote should not be fully repressible. The results obtained with one of the $C$ mutants (C1) in Table 2 show that repression is partially blocked by this mutation, while with another mutant (C2) repression is normal. The behavior of the $C 1$ mutant proves that a $C$ mutation and an $\mathrm{R1}^{-}$constitutive mutation can affect the same function required for repression, and therefore that both mutations belong to a common cistron, which is designated as $R 1$.

Summary of phenotypic effects of mutations in the R1 gene: Several different types of $R 1$ mutants are now known, as described in the present and previous reports. ${ }^{7,} 9$ These can be classified into the three general types listed in Table 3, according to

TABLE 3

Phenotypic Characteristics of $R 1$ Mutants

\begin{tabular}{lcc}
\multicolumn{3}{c}{ PhenOTYPIC ChaRACTERISTICs OF R1 MUtaNTs } \\
Induction of phosphatase & $\begin{array}{c}\text { Repression of phosphatase } \\
\text { synthesis }\end{array}$ \\
Wild-type strain & normal & normal \\
Mutant type 1 & normal & defective \\
Mutant type 2 & defective & normal \\
Mutant type 3 & defective & defective
\end{tabular}

the effects of the mutations on induction and repression of alkaline phosphatase. The different types were originally isolated as either constitutive mutants (type 1) or phosphatase-negative mutants (type 2) or both (type 3). Of particular importance is the fact that a mutant type 3 can be produced by a single site (revertible) mutation, showing that both the functions of induction and repression can be affected by a mutational event at one genetic site.

Discussion.-By analyzing the behavior of a number of different $R 1$ mutants, as summarized in Table 3, it has been possible to demonstrate the control exercised by the $R 1$ gene over two regulatory functions, those of induction and repression of alkaline phosphatase synthesis. An explanation for this dual role of the $R 1$ gene has been proposed (see the introductory section) which postulates that the product of the $R 1$ gene is an inducer of alkaline phosphatase and that this inducer also serves 
as the precursor for a repressor of alkaline phosphatase. In this way, we can account for the fact that a mutation in the $R 1$ gene can block the synthesis of the enzyme (despite the presence of a normal $P^{+}$structural gene) or block repression of enzyme synthesis or do both. At present, we have no information about the chemical nature of such an inducer or the site of its action. In line with present ideas about protein synthesis, the requirement for an inducer might involve either the formation of messenger RNA for alkaline phosphatase or the functioning of messenger RNA as a template in alkaline phosphatase synthesis. It follows that the role of the repressor can be interpreted as that of a competitive inhibitor of the inducer.

Summary.-Certain mutations in the $R 1$ regulatory gene for alkaline phosphatase completely prevent enzyme synthesis despite the presence of a normal $P^{+}$structural gene. An $F^{\prime}$ heterozygous strain that carries this mutation on the chromosome and a $P^{-}$mutation on the episome $\left(R 1^{+} P^{-} / R 1^{-} P^{+}\right)$produces a normal level of enzyme. This observation supports the proposal that the $R 1$ gene specifies the formation of an endogenous inducer required for alkaline phosphatase synthesis. The inducer appears to function also as the precursor of a repressor for alkaline phosphatase.

We wish to express our gratitude to Mrs. Suzanne Garen for her participation in these experiments.

* The work was supported by grants from the National Science Foundation and National Institutes of Health (U.S. Public Health Service).

${ }^{1}$ Levinthal, C., Brookhaven Symp. Biol., 12, 76 (1959).

2 Garen, A., in Microbial Genetics (Cambridge University Press, 1960.)

${ }^{3}$ Garen, A., C. Levinthal, and F. Rothman, J. Chimie Physique, 58, 1068 (1961).

${ }^{4}$ Horiuchi, 'T., S. Horiuchi, and D. Mizuno, Nature, 183, 1529 (1959).

5 Torriani, A., Biochim. Biophys. Acta, 38, 460 (1960).

${ }^{6}$ Garen, A., and C. Levinthal, Biochim. Biophys. Acta, 38, 470 (1960).

${ }^{7}$ Echols, H., A. Garen, S. Garen, and A. Torriani, J. Mol. Biol., 3, 425 (1961).

8 Jacob, F., and J. Monod, J. Mol. Biol., 3, 318 (1961).

${ }^{9}$ Garen, A., and H. Echols, J. Bacterol., 83, 297 (1962).

${ }^{10}$ Garen, A., and S. Garen, in preparation.

11 Jacob, F., and E. A. Adelberg, C. R. Acad. Sci. (Paris), 249, 189 (1960).

\section{ON THE AGREEMENT OF AMINO ACID REPLACEMENT DATA WITH CODE DESIGNATIONS FOR THE AMINO ACIDS}

\section{By Richard W. Hendler}

LABORATORY OF CELLULAR PHYSIOLOGY AND METABOLISM, NATIONAL HEART INSTITUTE

Communicated by Robert J. Huebner, June 20, 1962

On the basis of specific responses to added nucleotide polymers in an acellular $E$. coli preparation, a nucleotide triplet code to represent the 20 common amino acids in living systems has been deduced. ${ }^{1,2}$ There is reason to believe that this code may actually be operative in nature in the translation from nucleic acid to peptide sequences. It is because of the attractiveness and importance of this idea that one must be careful in evaluating the evidence accrued to support this concept. 